\title{
Pressure-induced Structural and Optical Transitions in Luminescent Bulk Cs4 $\mathrm{PbBr}_{6}$
}

W. C. Ferreira a, B. S. Araújo ${ }^{\text {a }}$, M. A. P. Gómez ${ }^{\text {a }, ~ F . ~ E . ~ O . ~ M e d e i r o s ~}{ }^{\mathrm{a}}$, C. W. A. Paschoala ${ }^{\mathrm{a}}$, C. B. da Silva ${ }^{a}$, P. T. C. Freire ${ }^{a}$, U. F. Kaneko ${ }^{b}$, F.M. Ardito ${ }^{b}$, NM Souza-Neto $^{\text {b }}$, and A. P. Ayala, ${ }^{a} *$

${ }^{a}$ Federal University of Ceará, Physics Department, Fortaleza, Ceará 65455-900, Brazil

b Brazilian Synchrotron Light Laboratory (LNLS), Brazilian Center for Research in Energy and Materials (CNPEM), Campinas, São Paulo 13083-970, Brazil. 


\section{Single-crystal $x$-ray diffraction}

The crystalline structure was confirmed using a Bruker D8 VENTURE single crystal diffractometer equipped with a Photon II detector and monochromatic Mo K $\alpha$ radiation $(\lambda=0.71073 \AA)$. A suitable crystal was chosen and mounted on a kapton fiber using a MiTeGen MicroMount. The data was indexed and integrated using SAINT included in the APEX3 software. The structure was solved using the SHELXT, and refinement by SHELXL under OLEX ${ }^{2}$.

Table S1: Crystal data and structure refinement for $\mathrm{Cs}_{4} 4 \mathrm{PbBr}_{6}$

\begin{tabular}{|c|c|}
\hline Empirical formula & $\mathrm{Cs}_{4} \mathrm{PbBr}_{6}$ \\
\hline Formula weight & 1218.34 \\
\hline Temperature/K & 110.47 \\
\hline Crystal system & trigonal \\
\hline Space group & $R \overline{3} c$ \\
\hline $\mathrm{a} / \AA$ & $13.7033(5)$ \\
\hline $\mathrm{c} / \AA ̊$ & $17.3358(9)$ \\
\hline Volume $/ \AA^{3}$ & 2819.2(3) \\
\hline $\mathrm{Z}$ & 5.99976 \\
\hline$\rho_{\text {calc }} \mathrm{g} / \mathrm{cm}^{3}$ & 4.306 \\
\hline$\mu / \mathrm{mm}^{-1}$ & 29.332 \\
\hline $\mathrm{F}(000)$ & 3072.0 \\
\hline Crystal size $/ \mathrm{mm}^{3}$ & $0.247 \times 0.226 \times 0.158$ \\
\hline Radiation & $\operatorname{MoK} \alpha(\lambda=0.71073)$ \\
\hline \multicolumn{2}{|c|}{$2 \Theta$ range for data collection $/{ }^{\circ} 5.82$ to 69.898} \\
\hline Index ranges & $-22 \leq \mathrm{h} \leq 21,-19 \leq \mathrm{k} \leq 22,-27 \leq 1 \leq 27$ \\
\hline Reflections collected & 17359 \\
\hline Independent reflections & $1353\left[R_{\text {int }}=0.0968, R_{\text {sigma }}=0.0525\right]$ \\
\hline Data/restraints/parameters & $1353 / 0 / 20$ \\
\hline Goodness-of-fit on $\mathrm{F}^{2}$ & 1.122 \\
\hline Final $R$ indexes $[\mathrm{I}>=2 \sigma(\mathrm{I})]$ & $\mathrm{R}_{1}=0.0608, \mathrm{wR}_{2}=0.1426$ \\
\hline Final $\mathrm{R}$ indexes [all data] & $\mathrm{R}_{1}=0.0795, \mathrm{wR}_{2}=0.1596$ \\
\hline \multicolumn{2}{|c|}{ Largest diff. peak/hole / e $\AA^{-3} 3.00 /-2.67$} \\
\hline
\end{tabular}


Table S2: Fractional atomic coordinates and equivalent isotropic displacement parameters $\left(\AA^{2} \times 10^{3}\right)$ for $\mathrm{Cs}_{4} \mathrm{PbBr}_{6}$. $\mathrm{U}_{\text {eq }}$ is defined as $1 / 3$ of the trace of the orthogonalised $\mathrm{U}_{\mathrm{IJ}}$ tensor.

\begin{tabular}{lrrrr}
\hline Atom & $\boldsymbol{x}$ & \multicolumn{1}{c}{$\boldsymbol{y}$} & $\boldsymbol{z}$ & $\mathrm{U}(\mathbf{e q})$ \\
\hline $\mathrm{Pb} 1$ & 0 & 0 & 0 & $31.4(2)$ \\
$\mathrm{Cs} 1$ & 0.33333 & $0.29173(7)$ & 0.91667 & $59.5(5)$ \\
$\mathrm{Cs} 2$ & 0 & 0 & 0.25 & $45.0(3)$ \\
$\mathrm{Br} 1$ & $0.69563(10)$ & $0.52579(9)$ & $0.23219(6)$ & $44.5(3)$ \\
\hline
\end{tabular}
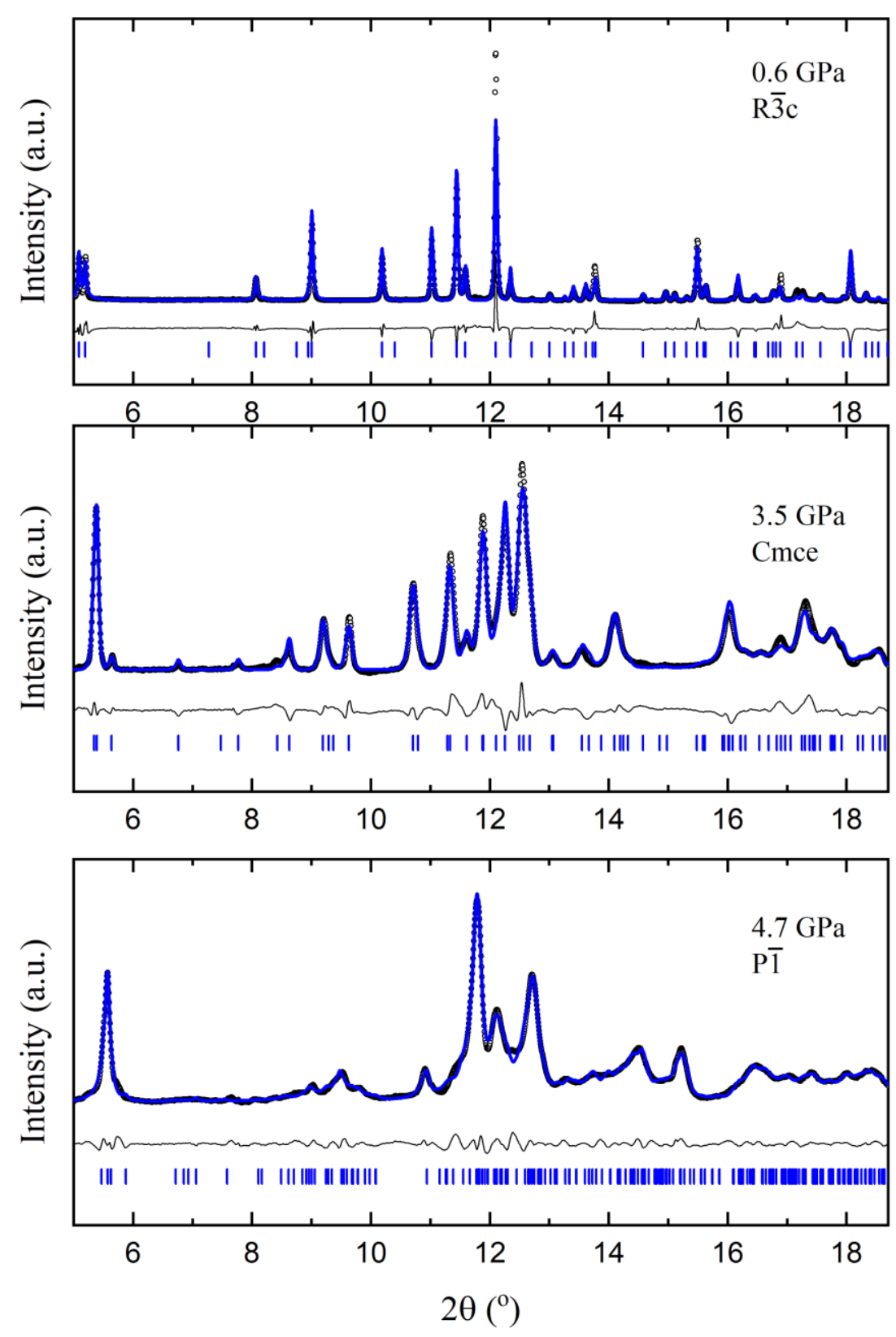

Figure S1. Rietveld refinement of $\mathrm{Cs}_{4} \mathrm{PbBr}_{6}$ at selected hydrostatic pressures. 
Table S3: Crystal structure of phases I, II and III of $\mathrm{Cs}_{4} \mathrm{PbBr}_{6}$

Pressure: 0.6 GPa

$$
\mathrm{a}=13.6756(5) \AA, \mathrm{c}=17.2917(10) \AA
$$

Volume: 2800.66(20) $\AA^{3}$, Space group: $R \overline{3} c \quad \mathrm{Z}: 6$

\begin{tabular}{cccc}
\hline $\mathrm{Pb}$ & 0 & 0 & 0 \\
$\mathrm{Cs} 1$ & $0.2982(5)$ & 0.3333 & 0.0833 \\
$\mathrm{Cs} 2$ & 0 & 0 & 0.25 \\
$\mathrm{Br} 1$ & $0.0250(7)$ & $0.1905(7)$ & $0.3980(4)$ \\
\hline
\end{tabular}

Pressure: 3.5 GPa

$$
\mathrm{a}=13.2887(20) \AA, \mathrm{b}=12.6058(20) \AA, \mathrm{c}=9.5105(20) \AA
$$

Volume: 1593.2(5) $\AA^{3}$, Space group: Cmce Z: 4

\begin{tabular}{cccc}
\hline $\mathrm{Pb}$ & 0 & 0 & 0 \\
$\mathrm{Cs} 1$ & 0.25 & $0.6042(6)$ & 0.25 \\
$\mathrm{Cs} 2$ & 0 & $0.3105(6)$ & $0.1133(8)$ \\
$\mathrm{Br} 1$ & 0 & $0.5688(7)$ & $0.1999(8)$ \\
$\mathrm{Br} 2$ & $0.3390(4)$ & $0.351(6)$ & $0.09209(7)$ \\
\hline
\end{tabular}

$$
\mathrm{a}=8.7152(20) \AA, \mathrm{b}=8.7635(20) \AA, \mathrm{c}=9.4070(20) \AA \text {, }
$$

Pressure: 4.7 GPa $\quad \alpha=91.78(1)^{\circ}, \beta=90.07(1)^{\circ}, \gamma=92.51(1)^{\circ}$

Volume: 718.1 (2) $\AA^{3}$, Space group: $P \overline{1} \quad Z: 2$

\begin{tabular}{cccc}
\hline $\mathrm{Pb} 1$ & 0 & 0 & 0 \\
$\mathrm{~Pb} 2$ & 0 & 0 & 0.5 \\
$\mathrm{Cs} 1$ & $-0.043(5)$ & $0.134(4)$ & $-0.182(3)$ \\
$\mathrm{Cs} 2$ & $0.212(6)$ & $0.299(3)$ & $0.429(4)$ \\
$\mathrm{Cs} 3$ & $0.309(5)$ & $0.1632(30)$ & $-0.094(4)$ \\
$\mathrm{Cs} 4$ & $0.725(5)$ & $0.419(4)$ & $0.263(4)$ \\
$\mathrm{Br} 1$ & $0.603(5)$ & $0.338(4)$ & $-0.003(6)$ \\
$\mathrm{Br} 2$ & $0.610(8)$ & $0.390(5)$ & $0.484(6)$ \\
$\mathrm{Br} 3$ & $0.583(5)$ & $0.296(5)$ \\
$\mathrm{Br} 4$ & $0.038(8)$ & $0.142(5)$ & $0.190(5)$ \\
$\mathrm{Br} 5$ & $0.544(7)$ & $0.242(4)$ & $0.050(6)$ \\
$\mathrm{Br} 6$ & $-0.034(6)$ & $0.022(7)$ & $0.525(5)$ \\
\hline
\end{tabular}




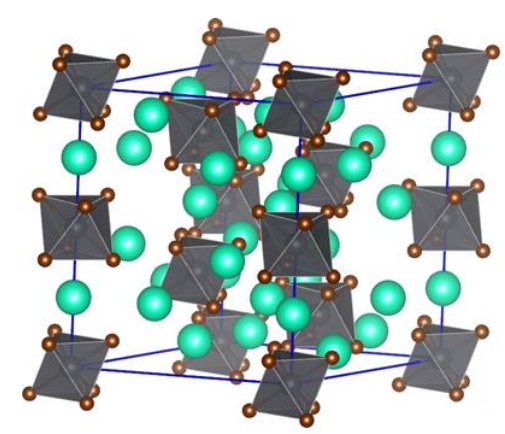

(a)

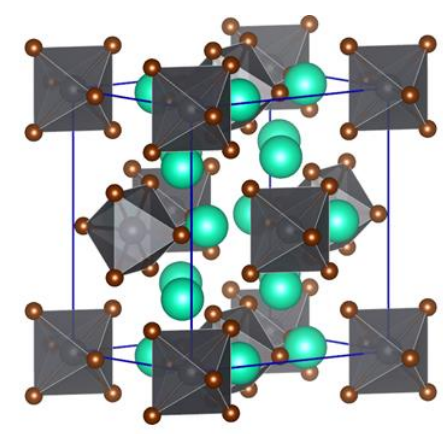

(b)

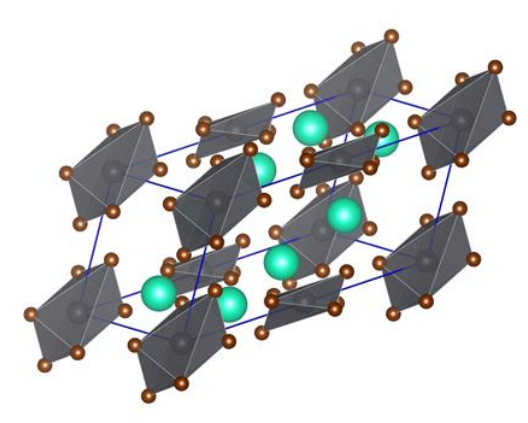

(c)

Figure S2. Crystal structures of phases (a) I, (b) II, and (c) III.

Table S4: Correlation table for $\mathrm{Cs}_{4} \mathrm{PbBr}_{6}$. The degrees of vibrational freedom for a given site species $\gamma$ is given by the symbol $\mathrm{f}^{\gamma}$. The translational and rotational degrees of freedom of the $\left[\mathrm{PbBr}_{6}\right]^{4-}$ octahedra become translational and librational lattice modes in the crystal.

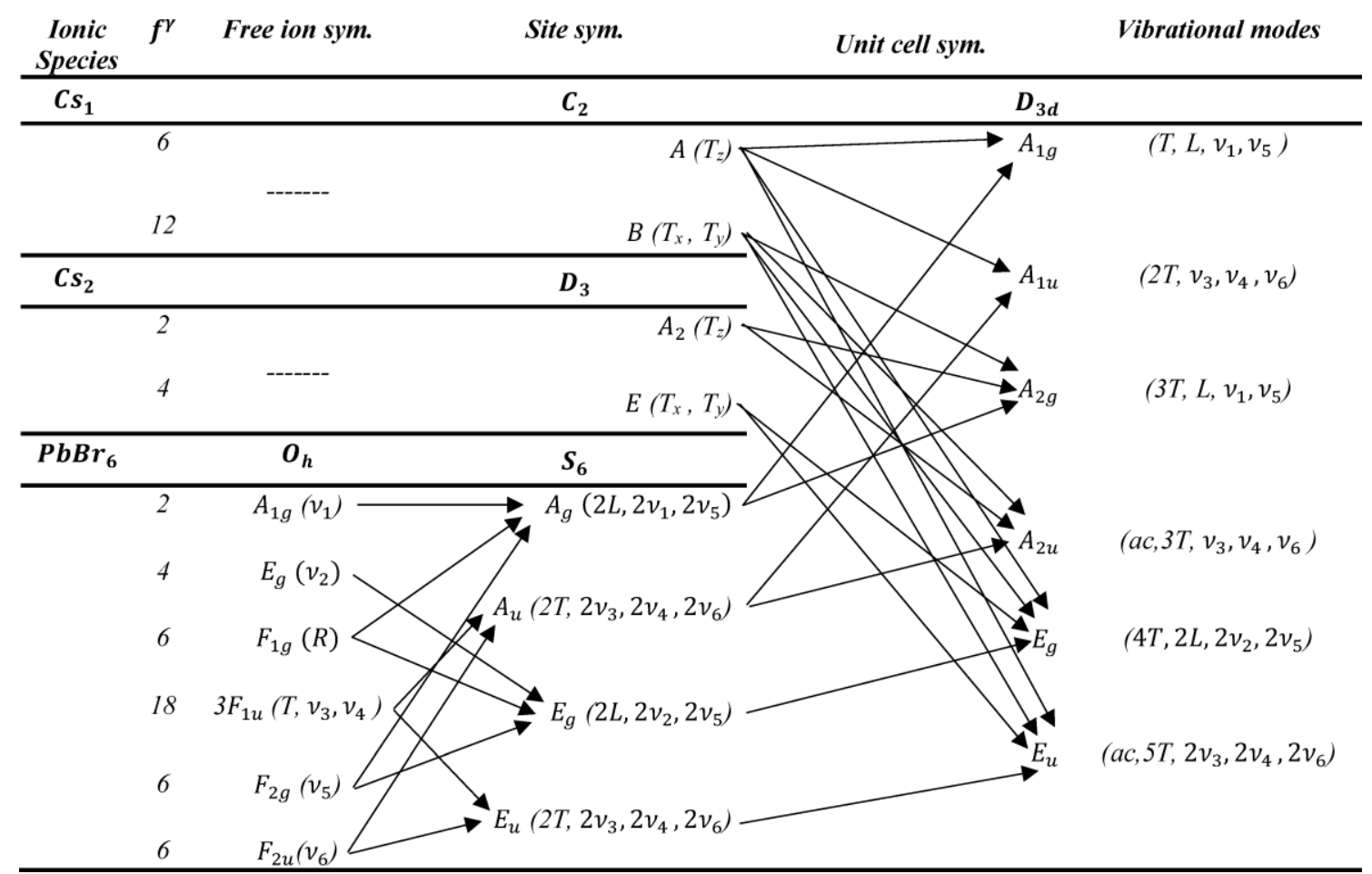

